\title{
Cooperación en la gobernanza sanitaria entre China y Argentina en el contexto de la COVID-19 desde la perspectiva de la gobernanza multinivel
}

\author{
Xiaoyu Ruan \\ Jingting Zhang
}

\author{
0184101118@shisu.edu.cn \\ Universidad de Estudios Internacionales de Shanghai \\ (SISU, China) \\ felisazhang007@gmail.com \\ Universidad de Estudios Internacionales de Shanghai \\ (SISU, China)
}

Recibido: 10/04/2021

Aceptado: 05/11/2021

Resumen: Este artículo tiene por objetivo analizar la cooperación en salud entre China y Argentina ante la crisis sanitaria de la COVID-19 desde la perspectiva de la gobernanza multinivel. Se ha demostrado que los actores estatales y subestatales desempeñaron un papel dominante en la primera etapa cooperativa, mientras que los actores no estatales empezaron a jugar un rol cada vez más activo y significativo en la segunda etapa. Mediante la cooperación sanitaria entre ambas naciones, Argentina ha logrado superar algunos desafíos traídos por la pandemia y ha mejorado la capacidad de gobernanza en salud frente al coronavirus. Para encarar la segunda ola de epidemia, existe la necesidad de profundizar la cooperación sanitaria entre los dos países a múltiples niveles.

Palabras clave: cooperación sanitaria; gobernanza sanitaria; cooperación Sur-Sur; gobernanza multinivel; relaciones sino-latinoamericanas

* Cómo citar: Ruan X. y Zhang J. (2021). Cooperación en la gobernanza sanitaria entre China y Argentina en el contexto de la COVID-19 desde la perspectiva de la gobernanza multinivel. Relaciones Internacionales, 30 (61), 141. https://doi.org/10.24215/23142766e141

Editor: Juan Alberto Rial, Instituto de Relaciones Internacionales Facultad de Ciencias Jurídicas y Sociales (Universidad Nacional de La Plata)

\section{Entidad editora: Relaciones}

Internacionales, es una publicación del Instituto de Relaciones Internacionales (Facultad de Ciencias Jurídicas y Sociales (Universidad Nacional de La Plata Argentina) 


\title{
Cooperation in health governance between China and Ar- gentina in the context of COVID-19 from the perspective of multilevel governance
}

\author{
Xiaoyu Ruan ${ }^{1}$, Jingting Zhang ${ }^{2}$
}

\begin{abstract}
This article aims to analyze the China-Argentina health cooperation in the face of the COVID-19 health crisis from the perspective of multilevel governance. It has been shown that state and sub-state actors played a dominant role in the first cooperative stage, while non-state actors started to play an increasingly active and significant role in the second stage. Through health cooperation between the two nations, Argentina has managed to overcome some of the challenges posed by the pandemic and has improved its health governance capacity in facing the coronavirus. To cope with the second wave of the epidemic, these two countries' health cooperation needs to be strengthened at multiple levels.
\end{abstract}

Keywords: health cooperation; health governance; South-South cooperation; multilevel governance; Sino-Latin American relations

\footnotetext{
1 Doctorando en Estudios de Área, Escuela de Relaciones Internacionales y Asuntos Públicos de la Universidad de Estudios Internacionales de Shanghai (SISU) . Magíster en Filología Hispánica, Universidad de Estudios Internacionales de Shanghai (SISU).

2 Doctora en Sociología, Universidad de Buenos Aires (UBA) . Magíster en Filología Hispánica, Universidad de Estudios Internacionales de Shanghai (SISU). Postdoctora y profesora, Universidad de Estudios Internacionales de Shanghai (SISU)
} 


\section{Introducción}

China y Argentina son amigos de confianza mutua y excelentes socios con una relación amistosa de larga historia. A inicios de 2020 cuando la pandemia de la COVID-19 se produjo en el país asiático, llegaron a China vuelos internacionales cargados de recursos sanitarios desde diversos países, entre ellos Argentina. Gracias a la asistencia internacional, las medidas restrictivas implementadas por el Gobierno chino, así como los esfuerzos del personal médico y toda la sociedad, la epidemia fue controlada a dos meses de su brote en el gigante asiático. Sin embargo, a partir de marzo, esta enfermedad contagiosa empezó a extenderse a nivel mundial. Mientras tanto China comenzó a reforzar la cooperación internacional en materia sanitaria para ayudar a otros países a enfrentar de manera conjunta el coronavirus, ya que para combatir de manera efectiva las enfermedades infecciosas como la COVID-19, se requiere una sólida cooperación mundial.

En el caso de Argentina, desde marzo de 2020 el país sudamericano entró en la etapa temprana de la epidemia. En ese momento, China fue el primer país que aportó apoyo internacional a Argentina en la lucha contra la COVID-19. Si bien en los primeros meses Argentina tuvo buenos resultados ante la crisis sanitaria comparada con otros países latinoamericanos, estaba frente a varios desafíos en plena pandemia, de modo que estrechó la cooperación sanitaria con China para contener la propagación de la enfermedad.

Este artículo divide la cooperación binacional en salud en dos etapas. La primera corresponde al periodo entre marzo y finales de junio de 2020, cuando el coronavirus estaba bajo control en el Estado sudamericano, y la segunda está comprendida entre finales de junio y la actualidad, en la que la epidemia está propagándose de manera acelerada por toda la Argentina. A partir del enfoque de la gobernanza multinivel, se procura analizar el papel que juegan los diversos actores, ya sean estatales, subestatales como no estatales, en la cooperación sanitaria entre los dos países en varios aspectos y sus contribuciones al reforzamiento de la capacidad de la gobernanza sanitaria de Argentina ante la pandemia.

En el siguiente apartado, se expone el marco teórico de la gobernanza multinivel en materia de salud. Después se analizan las necesidades de cooperación en salud entre ambas partes y las acciones realizadas por los múltiples actores involucrados en la gobernanza sanitaria durante las dos etapas. Para terminar, se concluirá con el análisis de las características de la cooperación sanitaria entre China y Argentina, los logros que han conseguido en las dos etapas, así como la necesidad de profundizar la cooperación en el futuro.

\section{Marco teórico}

El concepto de "gobernanza" no tiene una definición uniforme ni unívoca en el mundo académico. Tradicionalmente, muchos expertos consideran el Gobierno como el único actor de la acción de la gobernanza. Según Daniel Kaufman (2005), la gobernanza se entiende como "las tradiciones e instituciones por las que se ejerce la autoridad en un país para el 
bien común"3 (Kaufman, 2005). De modo similar, de acuerdo con Francis Fukuyama (2003), la gobernanza se refiere a "la capacidad de un Gobierno para establecer y hacer cumplir las normas, y para prestar servicios, independientemente de que ese Gobierno sea democrático o no"4 (Fukuyama, 2003). Sin embargo, debido a la percepción de que la Administración tradicional no ha conseguido los objetivos esperados, hay expertos que empiezan a enfocarse en el rol de los actores no estatales en la gobernanza. Tal como señala R. A. W. Rhodes (2007), la gobernanza es más amplia que el Gobierno y abarca los actores no estatales, así que la define como "gobernar con y a través de redes"5 (Rhodes, 2007), las cuales se caracterizan por ser descentralizadas y dirigidas.

De la misma manera, en cuanto a la definición de gobernanza en salud, se destaca generalmente el rol de las autoridades. De acuerdo con la Organización Mundial de Salud (OMS) (2008), la gobernanza en el sector sanitario se entiende por "una amplia gama de funciones relacionadas con la dirección y la elaboración de normas que llevan a cabo los Gobiernos o tomadores de decisiones cuando tratan de alcanzar los objetivos de la política sanitaria nacional que conducen a la cobertura sanitaria universal" 6 . No obstante, en un contexto cada día más pluralista de la actualidad, han surgido una multitud de proveedores diferentes, tanto formales como informales, públicos y privados en el aérea sanitaria (Organización Mundial de la Salud, 2008), por lo que la OMS subraya que "más allá del sistema sanitario oficial, la gobernanza significa colaborar con otros sectores, incluidos el sector privado y la sociedad civil, para promover y mantener la salud de la población de forma participativa e inclusiva"7. En la misma línea, Armando Arredondo et al (2009) define la gobernanza en salud como "la manera en que [los actores] del ámbito de la salud interactúan para producir, distribuir y consumir el bien de salud en función de la demanda de servicios y las necesidades de salud a los usuarios". De ahí que hoy en día cuando se analiza la gobernanza sanitaria, hay que pasar del marco analítico unitario, que solo se centra en los actores estatales, a un marco multinivel, que no solamente da importancia a los Gobiernos, sino que valoran el rol colaborativo de los actores no estatales.

3 "[...] the traditions and institutions by which authority in a country is exercised for the common good." Traducción de los autores del artículo.

4 "[...] a government's ability to make and enforce rules, and to deliver services, regardless of whether that government is democratic or not." Traducción de los autores del artículo.

5 “[...] governing with and through networks." Traducción de los autores del artículo.

6 "[...] a wide range of steering and rule-making related functions carried out by governments/decisions makers as they seek to achieve national health policy objectives that are conducive to universal health coverage." Traducción de los autores del artículo. Véase: https://www.who.int/healthsystems/topics/stewardship/en/

7 "Beyond the formal health system, governance means collaborating with other sectors, including the private sector and civil society, to promote and maintain population health in a participatory and inclusive manner." Traducción de los autores del artículo. Véase: https://www.who.int/healthsystems/topics/stewardship/en/ 
El término de "gobernanza multinivel" fue planteado por el politólogo Gary Marks en 1993 para caracterizar la dinámica de toma de decisiones de la Unión Europea. Según Marks (1993), se refiere a "un sistema de negociación continua entre Gobiernos anidados en varios

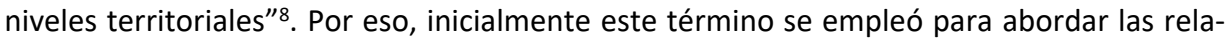
ciones intergubernamentales. Pero hoy en día, la gobernanza multinivel se utiliza para hacer referencia a la dispersión de la autoridad de manera vertical entre múltiples niveles de Gobiernos, como el local (subnacional), el nacional y el global (supranacional), y de manera horizontal entre diferentes sectores de interés y esferas de influencia, incluidos los actores no gubernamentales, mercados y sociedad civil ( (Daniell \& Kay, 2017) ).

En lo referente a la cooperación, de acuerdo con los académicos realistas de Relaciones Internacionales, se entiende como acciones gubernamentales realizadas en nombre de intereses nacionales y no por benevolencia (Kennan, 1971). Sin embargo, desde el punto de vista liberal institucionalista, se define a este término como una situación no necesariamente opuesta al conflicto, sino derivada de una compleja interdependencia y que necesita altos niveles de coordinación política (Keohane, 2005). Debido a que la cooperación se centra en el desarrollo internacional, se identifican dos formas de entendimiento acerca de tal acción: cooperación como ayuda y cooperación como asociación (Souza, 2014; Morvaridi \& Hughes, 2018). La primera forma corresponde a la Cooperación Norte-Sur, ofrecida por los países desarrollados a los en vías de desarrollo, que es una relación vertical y asimétrica, mientras que la segunda se refiere a la cooperación realizada entre países en desarrollo o Cooperación Sur-Sur (CSS), que tienden a ser una relación horizontal (Vadell et al, 2020). En este artículo, nos enfocamos en la CSS, ya que China y Argentina son dos países en desarroIlo. Según Naciones Unidas (2011), la CSS son las "actividades cooperativas entre los países en desarrollo sobre la base de solidaridad en una serie de áreas, incluyendo el comercio y la inversión, la cooperación financiera, técnica y tecnológica y el intercambio de conocimientos, experiencias, políticas y mejores prácticas." ${ }^{\prime 9}$ Autores como Rafael Domínguez Martín $(2016 ; 2017)$ y Michael Dunford (2020) han señalado que China se ha convertido en el principal promovedor de la CSS a partir del siglo XXI y ha desarrollado su propio estilo de cooperación basándose en los Cinco Principios de la Coexistencia Pacífica, que son el respeto mutuo por la soberanía y la integridad territorial, la no agresión mutua, la no interferencia en los asuntos internos de otros países, igualdad y beneficio mutuo, y la coexistencia pacífica.

Ante la creciente preocupación por la crisis sanitaria causada por la pandemia COVID19, el ámbito de la salud ha sido un tema cada día más relevante en la cooperación internacional en la actualidad, especialmente en la CSS. Siendo el mayor país en desarrollo y el primer país que se ha recuperado de la epidemia, China se ha ofrecido a cooperar con muchos países en desarrollo para combatir el coronavirus. Zhang Jie (2020) ha estudiado la

8 "[...] a system of continuous negotiation among nested governments at several territorial tiers." Traducción de los autores del artículo.

9 "[...] cooperation activities among the developing countries on the basis of solidarity in a number of areas, including

trade and investment, financial, technical and technological cooperation and the sharing of knowledge, experiences, policies and best practices." Traducción de los autores del artículo. 
cooperación en gobernanza sanitaria ante la COVID 19 entre China y el Sudeste Asiático, enfocándose en las dos formas cooperativas que se han desarrollado entre ambas partes. La autora destaca que China ha realizado la cooperación en materia de salud con los países de Asia Sudoriental, por un lado, de forma multilateral a través de la plataforma de ASEAN y simultáneamente de manera bilateral con los propios países, lo cual está en consonancia con el carácter regional del Sudeste Asiático, que es diverso y a la vez unificado, lo que aumenta la eficacia de la cooperación.

Zeng Aiping (2021) ha investigado la cooperación en gobernanza en salud entre China y África principalmente a partir del siglo XXI, desde la perspectiva de desarrollo conjunto de la "Ruta de la Seda Sanitaria", y ha mencionado los retos en la construcción de la Ruta y las estrategias de profundización de dicha iniciativa, recalcando que los seis decenios de cooperación médica y sanitaria y la lucha conjunta contra la COVID-19 entre ambas partes han proporcionado una buena base para impulsar la construcción de la "Ruta de la Seda Sanitaria". Asimismo, Xin Qiang y Wen Shaobiao (2020) han apuntado que la pandemia del coronavirus ha puesto de manifiesto la importancia y el valor estratégicos de la construcción de una "Ruta de la Seda Sanitaria", pero al mismo tiempo ha planteado nuevos tareas y desafíos. Los autores han resumido que a fin de promover la profundización y el desarrollo de la "Ruta de la Seda Sanitaria", China debe establecer un sistema de bioseguridad, mejorar la estructura de gobernanza multilateral internacional y potenciar la institucionalización de la misma Ruta. Por lo tanto, la construcción de la Ruta de Seda Sanitaria ha sido una manifestación concreta de la cooperación en la gobernanza sanitaria de China con los otros países en la actualidad y China está mostrando cada vez mayor voluntad e interés por estrechar la cooperación internacional en el sector de salud ante la pandemia.

Aparte de Asia y África, América Latina y el Caribe también son un escenario importante de la CSS impulsada por China (Domínguez Martín, 2016; 2017). En cuanto a la cooperación sanitaria entre ambas partes, el Gobierno chino ha explicitado este tema en los dos Documentos sobre la Política de China Hacia América Latina y el Caribe de 2008 y 2016, ${ }^{10}$ que proponen una asociación de cooperación integral con la región latinoamericana. Ambos documentos han destacado la cooperación en control de enfermedades, la colaboración de capacitación del personal médico y el suministro de materiales necesarios por parte de China para mejorar las

10 En el Documento de 2018 se expresa:" El Gobierno chino está dispuesto a promover activamente el intercambio y la colaboración médicos y sanitarios con los países latinoamericanos y caribeños, tomar el uno las experiencias del otro como referencia y desenvolver la cooperación en los ámbitos del control de enfermedad, tratamiento emergente de eventualidades sanitarias públicas y la prevención y control del SIDA y la gripe aviar, entre otros. Continuará enviando misiones médicas, a la vez de suministrar medicinas, aparatos y materiales médicos que necesiten las misiones médicas enviadas y ayudar a mejorar las instalaciones médicas y capacitar al personal médico de los países pertinentes." Véase en:

https://www.fmprc.gov.cn/esp/zxxx/t521035.shtml; en el Documento de 2016 se define la cooperación sanitaria como: "Ampliar el intercambio y la colaboración en los ámbitos de control de enfermedad, epidemia regional o mundial, tratamiento de emergencia de incidentes de salud pública, entre otros. China continuará ayudando a ALC en la capacitación del personal médico, mejorando sus instalaciones médicas y enviando misiones médicas, y está dispuesta a prestar asistencia, dentro de sus posibilidades, a ALC para la prevención y el control del brote repentino de enfermedades contagiosas." Véase en:

https://www.fmprc.gov.cn/esp/wjdt/wjzc/t1418256.shtml. 
instalaciones médicas de los países de América Latina y el Caribe. Cabe mencionar que se agregó al Documento de 2016 "la prevención y el control del brote repentino de enfermedades contagiosas", que ha sentado una base firme para la cooperación entre China y América Latina y el Caribe en la lucha contra la COVID-19.

A continuación, tomamos la cooperación en la gobernanza sanitaria entre China y Argentina como objeto de estudio y un ejemplo de la CSS de China con América Latina y el Caribe en materia de salud. Teniendo en cuenta todo lo que se ha mencionado anteriormente en este artículo, la gobernanza en salud desde la perspectiva de la gobernanza multinivel se refiere a la manera en que los actores tanto estatales como no estatales interactúan y colaboran entre sí para responder a la demanda y avanzar en la universalidad de los servicios de salud. Se usa el marco multinivel para analizar la cooperación sanitaria entre los dos países en el contexto de la pandemia de la COVID-19, a través de lo cual se demuestran los múltiples esfuerzos que han hecho los Gobiernos, empresas y la sociedad civil de ambos países en la provisión de insumos críticos, la protección al personal en la primera línea, el intercambio de experiencias antiepidémicas, así como la investigación sobre las vacunas.

\section{Antecedentes y necesidades de cooperación sanitaria entre China y Ar- gentina en la primera etapa de la lucha contra el coronavirus (desde marzo hasta finales de junio de 2020)}

La cooperación sanitaria entre China y Argentina puede remontar su origen a finales de la década de 1980. En 1988, durante la visita del entonces presidente argentino Raúl Ricardo Alfonsín a China, se concretaron los Acuerdos de Cooperación sobre la Antártida, Investigación y Aplicación de la Ciencia Astronáutica y Cuarentena Animal y Cooperación Sanitaria entre ambos países, los cuales iniciaron las primeras relaciones sanitarias entre el país asiático y el sudamericano. Después de la entrada en el siglo XXI, los dos países han intensificado la cooperación en el ámbito de la salud mediante varios convenios. ${ }^{11} \mathrm{~A}$ pesar de ello, el tema de prevención y tratamiento de enfermedades infecciosas emergentes solo se convirtió en una preocupación explícita con el brote de la pandemia de coronavirus en 2020. Además, la anterior cooperación sanitaria entre ambas naciones se desarrolló principalmente a nivel estatal; no contó con una participación activa de los Gobiernos locales y los actores no estatales. Es recién desde el marzo de 2020 que se ha visto incrementada la presencia de los Gobiernos subnacionales, las empresas y la sociedad civil en la gobernanza

11 En 2004, se firmó en Beijing el Convenio Marco de Cooperación en Materia de Salud y Ciencias Médicas; En 2013, se acordó la firma del Plan de Acción conjunta 2013-2016, que estaba destinado al desarrollo y fortalecimiento de la cooperación mutua en diversas áreas prioritarias del sector salud, tales como políticas de salud e investigación, control y prevención de enfermedades, cooperación en ciencias médicas y educación, y medicina tradicional china; En 2015, se subscribió el Convenio Marco para el Fortalecimiento de Cooperación en Materia de Salud y Ciencias Médicas; En 2018, se rubricó el segundo Plan de Acción Conjunta para el período 2019-2023, en el cual se abordó el tema de salud. 
sanitaria.

El primer caso confirmado de la COVID-19 en Argentina se dio a conocer el 3 de marzo de 2020 y se confirmó la primera muerte el 7 de ese mes. Durante el primer mes, los casos infectados aumentaron a un ritmo reducido, que pasaron de doce casos el 8 de marzo a 1.554 el 5 de abril. ${ }^{12}$ Sin embargo, a finales de abril los casos contagiados subieron de manera acelerada, superaron los 5000 el 5 de mayo y se dispararon hasta llegar a 10.649 el 22 de mayo. ${ }^{13}$ En cuanto a las cifras de mortalidad, se registraron más de 200 muertos a finales de abril y se acumularon más de 500 fallecidos un mes después. ${ }^{14}$ Por otra parte, en comparación con otros países latinoamericanos, en esta etapa temprana, Argentina logró mantener controlada la epidemia debido a las medidas restrictivas implementadas por el Gobierno e incluso se situó entre los países que mejor respondían a la pandemia del coronavirus elegidos por la revista estadounidense Time, siendo el único país latinoamericano que apareció en la lista (Télam, 2020b).

No obstante, en la etapa temprana, Argentina estaba frente a varios desafíos gigantescos en la lucha contra la pandemia. En primer lugar, se evidenció la falta de insumos necesarios para combatir la COVID-19. De acuerdo con los resultados arrojados de una encuesta realizada entre 1 y 3 de abril al personal de salud, la principal preocupación manifestada por los participantes fue la insuficiente disponibilidad de recursos institucionales -en particular de los equipos de protección personal acorde a las recomendaciones oficiales (Ortiz et al., 2020). En segundo lugar, debido a la escasez de elementos de protección, el personal de primera línea de combate contra el coronavirus estaba expuesto a altos riesgos de contagio en su trabajo. Por lo tanto, los médicos en Buenos Aires presentaron en abril un amparo ante la justicia por la escasez de material de protección para el personal de la salud en varios de los principales hospitales de la ciudad (CNN, 2020). A finales de mayo, los trabajadores de la salud representaron el 20 por ciento de los casos confirmados en la provincia de Buenos Aires (Diagonales, 2020). En tercer lugar, hacían falta recursos humanos profesionales y capacitados para los servicios sanitarios. Según la encuesta mencionada anteriormente, se observaron la demanda de lineamientos unificados para la formación y la necesidad de capacitaciones "a medida" para los diferentes sectores de la salud (Ortiz et al., 2020). Si bien los participantes de la encuesta habían asistido a capacitaciones, eso no implicó que

12 "Situación epidemiológica coronavirus-dengue- sarampión”, disponible en: https://www.argentina.gob.ar/sites/default/files/2020-03-08-situacion-epidemiologica.pdf; "Reporte diario vespertino / 05-042020" de informes diarios del Ministerio de Salud, disponible en: https://www.argentina.gob.ar/sites/default/files/05-04-20-reporte-vespertino-covid-19.pdf

13 “Reporte diario vespertino / 05-05-2020", disponible en: https://www.argentina.gob.ar/sites/default/files/05-05-20_reporte_vespertino_covid_19.pdf; "Reporte diario vespertino / 22-05-2020", disponible en: https://www.argentina.gob.ar/sites/default/files/22-05-20_reporte-vespertino_covid-19.pdf

14 “Reporte diario vespertino / 28-04-2020", disponible en: https://www.argentina.gob.ar/sites/default/files/28-04-20-reporte-vespertino-covid-19.pdf; "Reporte diario vespertino / 27-05-2020", disponible en: https://www.argentina.gob.ar/sites/default/files/27-05-20_reporte_vespertino_covid_19.pdf 
ellos se autopercibieran con las habilidades necesarias. Además, los profesionales de la enfermería no están bien distribuidos en Argentina. Hay ciudad o provincias, tales como Ciudad Autónoma de Buenos Aires, Jujuy y Neuquén, en donde existe una mayor concentración de profesionales, pero hay otras, como Formosa y Córdoba, en las que la ausencia de profesionales es muy marcada, ${ }^{15}$ lo cual podría provocar el colapso del sistema sanitario en algunas provincias ante la pandemia.

Mientras tanto, China ya estaba en la etapa de pospandemia y tenía la capacidad para ayudar a Argentina a atravesar los momentos difíciles en el combate contra la COVID-19. A mediados de marzo, gracias a las medidas restrictivas y los esfuerzos unidos y arduos del pueblo chino, el país asiático consiguió mantener bajo control la pandemia y estaba preparado para regresar a la normalidad paso a paso. El número de los nuevos casos de contagios locales en la China continental había descendido por debajo de 20 diarios a mediados de marzo (La Vanguardia, 2020). El personal sanitario que acudió de todas partes de China a Wuhan para luchar contra el coronavirus también comenzó a emprender el viaje de regreso (Cao, 2020). Tras casi dos meses de combatir la enfermedad, los trabajadores de la salud de China habían acumulado ricas experiencias en el tratamiento de la enfermedad, que podrían ser útiles para los médicos y enfermeros argentinos que recién empezaban a tratar a los pacientes infectados. Después de la escasez de insumos sanitarios en la etapa inicial, "la fábrica del mundo" ya estaba en condiciones de producir suficientes materiales de salud para cubrir las necesidades de toda la nación e incluso para exportarlos a otros países. Por ende, China estaba capacitada para ofrecer ayuda a Argentina para enfrentarse de manera conjunta con la crisis sanitaria.

Por otro lado, siendo el amigo de mutua confianza y buen socio comercial de Argentina, China tiene suficiente voluntad para echar una mano a Argentina cuando el país latinoamericano estaba encarando tantos retos en la lucha antiepidémica. Argentina es uno de los primeros países en América Latina que establecieron relaciones diplomáticas con China, que se iniciaron desde el año 1972. A partir de entonces, las frecuentes visitas mutuas de los dirigentes de los dos países, así como de misiones de diferentes niveles han fomentado continuamente los lazos binacionales. En los últimos años, con el acercamiento de las relaciones comerciales entre China y Argentina, la presencia del gigante asiático se ha vuelto cada vez más prominente en el país sudamericano. Desde 2019, China ha sido el principal comprador de productos agropecuarios argentinos y en junio de 2020 se convirtió en el primer socio comercial del país austral, desplazando a Brasil (Télam, 2019; 2020a). Hoy en día, más de 80 empresas chinas cuentan con sucursales acreditadas en Argentina, entre las cuales se destacan el Banco Industrial y Comercial de China, Banco de China, Sinopec y China National Offshore Oil Corporation. ${ }^{16}$ Al mismo tiempo, la cantidad de inmigrantes de procedencia china en la Argentina ha experimentado un aumento robusto en los recientes años. Según el ex embajador chino en Argentina, Yang Wanming, en 2016 había cerca de 180.000 inmigrantes chinos en país sudamericano y en 2018 la cifra estimada aclanzó los 200.000

15 Datos sobre fuerza de trabajo, véase: https://www.argentina.gob.ar/salud/observatorio/datos/fuerzadetrabajo

16 Véase: http://www.mofcom.gov.cn/dl/gbdqzn/upload/agenting.pdf 
(Ng y Restivo, 2018). Las relaciones comerciales estrechas con Argentina y los vínculos con la comunidad china en el territorio argentino constituyen buenas razones para que China esté junto al país sudamericano para afrontar el coronavirus. Además, el hecho de establecer una cooperación internacional en materia sanitaria ante la epidemia puede ser considera una buena práctica del concepto "comunidad de destino común de la humanidad", planteado por el presidente chino Xi Jinping, especialmente cuando ningún país puede sentirse a salvo de esta crisis global sanitaria.

En resumen, si bien Argentina dio una buena respuesta en la etapa temprana de la lucha contra la epidemia, el país se encontraba en medio de varios problemas urgentes. A su vez, China ya estaba en el camino hacia la recuperación de la pandemia. Teniendo en cuenta las largas y amistosas relaciones diplomáticas y los estrechos lazos comerciales entre ambas partes, la presencia significativa de la comunidad china en Argentina, así como la búsqueda de China de una comunidad de destino común de la humanidad, el país asiático mostró buena disposición a cooperar con Argentina en el combate contra el coronavirus. Por consiguiente, China era el primer y único país que ofreció asistencia internacional a Argentina a finales de marzo de 2020. Tras numerosas conversaciones e intercambios entre el presidente chino Xi Jinping y su par argentino, Alberto Fernández, se estableció una cooperación binacional destinada a combatir la pandemia de la COVID-19 (Niebieskikwiat, 2020).

\section{Cooperación en gobernanza sanitaria entre China y Argentina en la pri- mera etapa}

A partir de finales de marzo de 2020, en el marco de cooperación binacional en materia sanitaria se realizaron varios esfuerzos principalmente a nivel estatal y subestatal, y con una participación coordinada de los actores no estatales.

\section{i. Cooperación en provisión de recursos sanitarios}

Ante la escasez de insumos críticos para enfrentar la COVID-19, los actores estatales implementaron varias medidas para sostener la provisión continua entre China y Argentina. Una acción de gran relevancia fue la construcción de un puente aéreo sin precedente entre los dos países, que conectaba la ciudad de Buenos Aires y la ciudad china de Shanghai, con escala en Auckland (Xinhua, 2020b). A través de esta ruta aérea, se llevó a cabo un total de 35 operaciones entre los meses de abril y julio de 2020, y se permitió el transporte de 887 toneladas de materiales e insumos médicos desde China a Argentina (Xinhua, 2020e). Entre ellos, no solo se encontraban las donaciones de país asiático, sino también las compras que hicieron las autoridades argentinas. A modo de ejemplo, nueve vuelos transportaron las 400 toneladas de insumos médicos que compró Kicillof, el gobernador de la provincia de Buenos Aires, por 10 millones de dólares (Ayerdi, 2020).

En cuanto a las donaciones, en su mayoría, procedieron de los ministerios centrales y los Gobiernos subnacionales que tienen relación de hermanamiento con las provincias o 
ciudades argentinas. ${ }^{17}$ Por ejemplo, a nivel ministerial, el Ministerio de Seguridad Pública de China hizo una donación de elementos sanitarios al Ministerio de Seguridad de Argentina a finales de mayo de 2020, los cuales se repartirían entre las fuerzas de seguridad dedicadas a la prevención del coronavirus, incluidas la Policía Federal Argentina, la Prefectura Naval Argentina, la Gendarmería Nacional y la Policía de Seguridad Aeroportuaria (Ibid.), ya que además de los trabajadores de la salud, las fuerzas de seguridad forman parte del personal de primera línea en combate contra el coronavirus. Asimismo, el Ministerio de Defensa de Argentina recibió un lote de materiales sanitarios donados por su par chino a mediados de junio de 2020 (Larre, 2020), que también contribuían a la protección de los agentes armados de la primera línea en zonas peligrosas.

A nivel subnacional, a mediados de mayo de 2020, llegó a la provincia de Mendoza una donación de 60 mil barbijos provenientes de Guangdong, provincia china que estableció relaciones hermanas con Mendoza en 2013 (ProMendoza, 2020). A principios de junio, la Municipalidad de Rosario recibió una valiosa donación de materiales sanitarios de la ciudad hermana china de Shanghai, incluidos 3.000 barbijos modelo N95, 300 trajes de protección médica y 20.000 barbijos quirúrgicos, que serían distribuidos entre los profesionales de la salud (Rosario Noticias, 2020a). Hasta finales de junio, al menos seis provincias o ciudades chinas enviaron toneladas de insumos sanitarios a Argentina, aparte de los dos Gobiernos locales indicados, incluyendo Shenzhen, Sichuan, Chengdu y Chongqing (Dinatale, 2020b).

Entre los actores no estatales, fueron las empresas multinacionales las que jugaron un papel prominente en el suministro de elementos sanitarios al pueblo argentino en la etapa temprana. A mediados de abril, 75 empresas chinas prepararon donaciones de respiradores, kits de detección del virus, barbijos y mamelucos, así como asistencia para su logística (Dinatale, 2020a). Huawei y Alibaba se encuentran entre las primeras empresas chinas que dieron apoyo internacional a Argentina. A comienzos del brote de la pandemia en marzo, antes de la llegada de la primera donación del Gobierno chino, la empresa Huawei donó a la Dirección Nacional de Migraciones de Argentina dos cámaras térmicas para detectar la fiebre de pasajeros que ingresaban en el aeropuerto de Ezeiza y posteriormente envió equipos de conectividad para los ocho hospitales en construcción, así como 100.000 barbijos y 15.000 trajes protectores (Miranda, 2020; Dinatale, 2020a). Poco después de la primera donación del Gobierno chino, la Fundación de Jack Ma y la Fundación de Alibaba enviaron una donación de 100 mil barbijos de uso médico, 50 mil kits de prueba y 10 ventiladores al país sudamericano. ${ }^{18}$

Hasta finales de junio, Argentina recibió apoyo internacional de una totalidad de más de 12 países y regiones, entre los cuales, China lleva la delantera en materia de donaciones (Dinatale, 2020b). Los materiales donados por China aliviaron en gran medida la escasez de insumos sanitarios en Argentina y aseguran la protección del personal que lucha en primera línea de batalla con el coronavirus. Hasta mediados de junio, en la provincia de Buenos Aires

17 De acuerdo con la Cancillería Argentina, hasta el julio de 2020, 17 ciudades y provincias argentinas han establecido 20 acuerdos de hermanamiento con sus pares chinos.

18 Véase: http://ar.chineseembassy.org/chn/sgxw_1/t1759050.htm 
el porcentaje de contagios entre el personal sanitario bajó de forma significativa, correspondiendo al 9, 7 por ciento del total de casos acumulados en la provincia.

\section{ii. Cooperación en el intercambio de experiencias contra la COVID-19}

Aparte de la cooperación en la provisión de recursos críticos para la lucha contra la epidemia, China y Argentina trabajaron de manera dinámica en el intercambio de experiencias antiepidémicas mediante numerosas videoconferencias. En general estos eventos virtuales son producto de una serie de esfuerzos multiniveles. Fueron promovidos y organizados por la Embajada China en Argentina y los diferentes niveles de Gobiernos chinos y argentinos, y contaron con la participación de funcionarios de las Administraciones estatales y locales competentes en el combate contra la COVID-19, expertos o trabajadores de la salud, así como representantes de organizaciones internacionales.

En los encuentros transpacíficos, se abordaron diversos temas críticos para la batalla contra la pandemia. Por un lado, se intercambiaron las medidas eficaces de prevención, control y diagnóstico, tales como la estricta prevención de casos de contagio procedentes del extranjero, la prevención y el control en la comunidad, el rastreo de personas de contacto cercano, el control del contagio en los hospitales, entre otros. Por el otro, se enfocan en las medidas de tratamiento y cuidado a los pacientes infectados, incluidos el uso y la combinación de medicamentos para los casos confirmados, el tratamiento de pacientes mayores de edad con enfermedades básicas y de mujeres embarazadas, así como los planes de tratamiento diferenciados para casos leves y graves.

Desde marzo hasta finales de junio, tuvieron lugar varias conferencias importantes entre ambas partes, en las que participaron diferentes actores multiniveles. El 20 de marzo por la noche, se organizó una videoconferencia entre una docena de autoridades del Ministerio de Salud de Argentina y expertos médicos chinos de la provincia oriental de Zhejiang con el objetivo de conocer mejor la experiencia china en el combate contra la epidemia de COVID-19 (Xinhua, 2020a). El 9 de abril por la mañana, los funcionarios de la provincia de Santa Fe realizaron una videoconferencia con sus homólogos y expertos chinos de la provincia hermana de Jiangsu para recibir recomendaciones y aprendizajes de sus pares chinos (Prensa Gobierno de Santa Fe, 2020). Asimismo, las autoridades sanitarias de la provincia de Santa Cruz y equipo médico de la UTE Represas Patagonia dialogaron el 21 de abril con sus pares chinos de la provincia de Yichang a través de un encuentro virtual (La Opinión Austral, 2020). Además de dichas provincias y ciudades chinas, el Gobierno de la ciudad capital de Beijing ${ }^{19}$, la Federación de la Juventud de China ${ }^{20}$ y la Cadena Global de Televisión de China ${ }^{21}$, entre otros actores estatales y no estatales, celebraron conferencias en línea con autoridades y expertos argentinos.

19 Véase: http://wb.beijing.gov.cn/en/sister_cities/other_cities/others_latest_news/202008/t20200818_1985522.html

20 Véase: https://www.unraf.edu.ar/index.php/noticias/1323-noticia-513

21 Véase: http://m.news.cctv.com/2020/06/13/ARTI30KJj0JLjf2vqAxVd8mB200613.shtml 
A través de los encuentros virtuales, se ha facilitado e intensificado el intercambio de experiencias, aprendizajes y conocimientos adquiridos por parte de las autoridades y los expertos médicos chinos en las prácticas antipandémicas con sus homólogos argentinos. Por lo demás, los profesionales argentinos de diversas partes del país pueden transmitir sus consultas de acuerdo con la situación y la necesidad locales con sus homólogos chinos, de tal forma que reciban consejos más específicos y apropiados para combatir la COVID-19.

\section{Necesidades de cooperación sanitaria entre China y Argentina en la se- gunda etapa de la lucha contra el coronavirus (desde finales de junio de 2020 hasta el presente)}

Debido a la buena respuesta de Argentina a la pandemia en los primeros dos meses, en un anuncio del 8 de mayo de 2020, el presidente Fernández dispuso el fin de la cuarentena para todo el territorio del país. Sin embargo, un mes después, el 9 de junio, Argentina por primera vez registró más de 1.000 nuevos casos infectados por el coronavirus ${ }^{22} y$ desde entonces, creció de forma sustancial el número de los nuevos contagios. El 1 de julio fueron confirmados 2.667 nuevos casos infectados y con estos registros se acumularon 67.197 casos positivos en el país sudamericano. ${ }^{23}$ Por lo tanto, volvieron a ser restrictivas las medidas de aislamiento social. Aun cuando el Gobierno argentino realizó varias prórrogas de la cuarentena, el 26 de agosto, se contabilizaron por primera vez más de 10 mil nuevas infecciones por la COVID-19 en Argentina y a siete meses del inicio de la cuarentena, el 19 de octubre, el país ya había llegado al millón de casos positivos de coronavirus. ${ }^{24}$ Por eso, a partir de finales de junio, Argentina empezó a enfrentarse con más desafíos en comparación con los primeros cuatro meses de la batalla contra el coronavirus.

En primer lugar, la escasez de insumos sanitarios seguía siendo una de las mayores preocupaciones, como lo había sido en la primera etapa. Hasta octubre, Argentina era uno de los países del mundo que menos tests por millón de habitantes había realizado desde el inicio de la pandemia. Estaba situada en el puesto 74 en una lista de 140 países sobre los cuales había datos para este indicador (Fitz Patrick y Crucianelli, 2020). En segundo lugar, se agudizó la pobreza en Argentina con el desarrollo de la pandemia. En el segundo semestre de 2020, la pobreza en el país llegó al 42 por ciento, lo que implica un fuerte incremento con respecto al 35, 5 por ciento del mismo período del 2019 (Lewkowicz, 2021). En consecuencia, cada vez había más familias a las que les faltaban no solo los materiales sanitarios sino también los recursos necesarios para sostener la vida. Por último, con el aumento acelerado de los casos confirmados, el sistema de salud de Argentina estaba al borde del colapso según

22 “Reporte diario vespertino / 09-06-2020", disponible en: https://www.argentina.gob.ar/sites/default/files/9-06-20-reporte-vespertino-covid-19.pdf

23 “Reporte diario vespertino / 01-07-2020", disponible en: https://www.argentina.gob.ar/sites/default/files/1-07-20_reporte_vespertino_covid_19.pdf

24 “Reporte diario vespertino / 26-08-2020", disponible en: https://www.argentina.gob.ar/sites/default/files/26-08-20-reporte-vespertino-covid-19.pdf; “Informes diarios. Reporte diario vespertino / 19-10-2020", disponible en: https://www.argentina.gob.ar/sites/default/files/19-10-20-reporte-vespertino-covid-19.pdf 
un comunicado emitido por la Sociedad Argentina de Terapia Intensiva (Esteban, 2020). Si bien el sistema de salud argentino aumentó en más del 40 por ciento la disponibilidad de unidades de terapia intensiva, a inicios de septiembre el Gobierno reconoció que la expansión del sistema de salud había llegado al límite.

A su vez, China logró mantener contenida la epidemia en el segundo semestre del 2020 y hasta la actualidad. Aunque se produjeron algunos rebrotes en varias ciudades y provincias, fueron controlados por los Gobiernos locales en poco tiempo. Debido a eso, China está volviendo a la normalidad del mundo previo a la pandemia y ha sido la única de las principales economías que creció el año pasado. Ante los nuevos y antiguos desafíos que afronta Argentina en la segunda etapa, China refuerza su cooperación en gobernanza sanitaria con el país sudamericano para fortalecer la capacidad del sistema sanitario argentino y aumentar su capacidad de resistencia a la epidemia. Además, se observa una participación cada vez más destacada de los actores no estatales en la segunda etapa en comparación con la primera.

\section{Cooperación en gobernanza sanitaria entre China y Argentina en la se- gunda etapa}

\section{i. Cooperación en el suministro de recursos sanitarios y materiales necesarios para la vida}

Al igual que la primera etapa, frente a la escasez de recursos sanitarios, China y Argentina profundizaron la cooperación sanitaria en el suministro de insumos necesarios para la batalla contra el coronavirus. A nivel estatal, se inició en noviembre, la segunda fase de las operaciones especiales aéreas para trasladar materiales críticos desde China a Argentina. En total, se realizaron siete vuelos entre Buenos Aires y Guangzhou en esta fase de operación. Como lo expresó el senador y ex canciller Jorge Taiana, esta cooperación tenía suma importancia para la provisión de equipamiento en Argentina y permitió que el país sudamericano tuviera una mejor preparación ante la pandemia y mejorara la capacidad del sistema sani$\operatorname{tario}^{25}$.

A nivel subnacional, más provincias y ciudades chinas se sumaron a la cooperación sanitaria. A modo de ejemplo, el 3 de julio de 2020 la municipalidad de Quilmes recibió un lote de materiales sanitarios donado por su ciudad hermana china, Nanchang. La donación incluía 28.000 barbijos descartables de uso médico y figuró en el marco de cooperación que hermana a ambas ciudades desde el año 2015 (El Sol, 2020). La asistencia sanitaria de Nanchang permitió al municipio argentino garantizar las medidas de prevención y cuidado de las personas más necesitadas en medio de la pandemia. Al mismo tiempo, la provincia china de Guizhou envió una donación de suministros médicos a la provincia hermana argentina de Jujuy (Xinhua, 2020c). Se trataba de un total de 9 mil mascarillas médicas donadas por el Hospital Popular Provincial a solicitud de la Secretaría de Integración Regional y Relaciones

25 Entrevista de Televisión Central de China con el señor Jorge Taiana, véase: http://www.xinhuanet.com/video/2020-07/06/c_1210688277.htm 
Internacionales de Jujuy en nombre del gobernador Gerardo Rubén Morales.

Con el fin de fortalecer el sistema de salud de Argentina, aparte de los insumos sanitarios, el Gobierno chino también donó al Ejército Argentino un nuevo hospital móvil en febrero de 2021. Era un centro de salud reubicable compuesto de 13 módulos con sus respectivos vehículos para transporte. El equipamiento contaba con un quirófano, laboratorio, radiología, planta de agua propia y otros servicios médicos. Además, disponía de cuatro carpas para sala de pacientes, 90 radios portátiles $\mathrm{VHF}^{26}$ y 15 radios de mochila VHF (Mary, 2020). La primera donación de esta magnitud por parte del país asiático fue en 2017 . Esta instalación no solo puede reforzar el sistema nacional de salud, ayudando al Ejército a realizar mejor sus actuales tareas contra la epidemia, sino que desempeñará un papel importante en la ejecución de las futuras operaciones de auxilio humanitario entre ambos países.

Cabe mencionar que, en la segunda etapa, los actores no estatales han jugado un papel importante en la provisión de insumos sanitarios y recursos necesarios para la vida, especialmente para las familias en situación de pobreza en Argentina. El 29 de junio, la Cámara de Comercio Chino de Rosario realizó una donación de más de 21 mil kilos de alimentos al Banco de Alimentos Rosario para la asistencia alimentaria de familias vulnerables (Rosario Noticias, 2020b). El presidente de la entidad, Chen Chunrong apuntó que la Cámara también había recibido un total de 2.325 .000 pesos de donaciones, los que se destinarían a la compra de recursos antiepidémicos, que se entregarían a los hospitales locales, la jefatura de policía, la fiscalía y el municipio. Este último, a su vez, se encargaría de distribuir los materiales a las instituciones y a las personas necesitadas. Asimismo, el 29 de julio, la Municipalidad de Córdoba recibió una donación de alimentos y materiales antiepidémicos de la Cámara de Comercio Chino de Córdoba, que había reunido una suma de 2.535 .000 pesos por parte de los chinos en la provincia. ${ }^{27}$

Las comunidades chinas que viven en Argentina también se ofrecieron a prestar asistencia material a sus vecinos argentinos. El 29 de noviembre de 2020, la Asociación de Chinos de Qingtian y el Equipo de Voluntarios Chinos en Argentina repartieron materiales sanitarios delante de la entrada del Supermercado Fuwang en el barrio Once de la Ciudad de Buenos Aires. En total, hicieron una donación de 40.000 barbijos descartables, 5.000 barbijos KN95 y 4.500 máscaras protectoras (NewsArgenChina, 2020a). El 4 de diciembre de 2020, el Equipo de Voluntarios Chinos en Argentina donó alimentos y materiales sanitarios a la fundación El Pobre de Asís y al comedor María Eva de la Comuna 13 de la Ciudad de Buenos Aires (NewsArgenChina, 2020b). Según la presidenta de la Junta Comunal 13, a partir del inicio de la pandemia, la comunidad china donó activamente alimentos y materiales a la Comuna 13 en reiteradas ocasiones, lo cual evidenció la estrecha relación entre la comunidad china y la población local ante la epidemia.

En resumen, en la segunda etapa cooperativa, tanto los actores estatales como los no estatales han aportado grandes esfuerzos para mitigar la ausencia de recurso sanitarios que

26 Abreviatura de inglés "very high frequency" (frecuencia muy alta) .

27 Véase: http://www.chinacelacforum.org/chn/zgtlmjlbgjgx/t1803502.htm 
afronta Argentina y ayudar a aquellas poblaciones más vulnerables como las familias que viven en situación de pobreza a mantenerse la vida ante el impacto del coronavirus.

\section{ii. Cooperación en el desarrollo y la investigación de vacunas}

Frente al aumento brutal de casos de contagio, varios países aceleraron los estudios de vacuna en 2020 , puesto que se considera la vacunación como la herramienta más efectiva contra la enfermedad infecciosa.

Argentina estaba entre los seis países en Latinoamérica que realizaban ensayos clínicos o planearon participar en alguno hasta diciembre de 2020 (Perez Sarmenti, 2021). El país sudamericano cuenta con varias ventajas al respecto. Por un lado, tiene una tradición de ensayos clínicos y larga experiencia en investigación. Por el otro, hay varias instituciones que garantizan la calidad de los ensayos. A modo de ejemplo, Argentina dispone de una agencia reguladora seria, la Administración Nacional de Medicamentos, Alimentos y Tecnología Médica (Anmat), que tiene la calificación de "Reference Authority" que le otorga la OMS y la Organización Panamericana de la Salud (OPS). Además, en el país también hay varios "contract research organizations", organizaciones profesionales cuya misión consiste en gestionar ensayos clínicos y que son de reconocido prestigio internacional.

Los ensayos clínicos de la vacuna requieren esfuerzos de colaboración por parte de múltiples actores, ya que hacen falta la autorización y supervisión del Gobierno, la ejecución e investigación de empresas farmacéuticas y organizaciones relativas, así como la participación de los voluntarios, por lo que constituye otro ejemplo típico de la gobernanza sanitaria multinivel.

Se aprobó en julio de 2020 el primer ensayo clínico de Fase III en Argentina (Ibid.). Un mes después, el 21 de agosto, el Gobierno Argentino autorizó realizar los ensayos clínicos de Fase III de vacuna inactivada china contra la COVID-19, lo cual marcó un hito histórico en la cooperación sanitaria entre ambas partes (Xinhua, 2020d). La vacuna fue desarrollada por la empresa china Sinopharm Group y los estudios se llevaron a cabo bajo la conducción de la Fundación Huésped, con la participación de 3.000 voluntarios (DW, 2021). A mediados de diciembre, la misma Fundación anunció el inicio de un nuevo estudio de Fase III de una vacuna de origen chino desarrollada por el Beijing Institute of Biotechnology y CanSino Biologics Inc, que era el segundo proyecto de ensayos clínicos de China aprobado en Argentina. Para el ensayo de la vacuna de CanSino solo buscaban reclutar 4.000 voluntarios, pero la cantidad de pedidos los desbordó, ya que había 20.000 personas anotadas para recibir la vacuna (Perez Sarmenti, 2021).

A fines de febrero de 2021, el Ministerio de Salud de Argentina dio luz verde al uso de emergencia de la vacuna china de la empresa Sinopharm, a partir de la recomendación de la Anmat (Ámbito, 2021). El 25 de febrero llegó a Argentina un cargamento de vacunas chinas con 904 mil dosis que estaban destinadas a la vacunación del personal docente de todo el país (Infobae, 2021). Por lo demás, tras una larga negociación con el Gobierno de Xi Jinping, Argentina ya había acordado la provisión de más de 20 millones de vacunas de procedencia china. Un mes después, el 26 de marzo, la Anmat recomendó a la ministra de Salud 
autorizar el uso de emergencia de la vacuna Sinopharm en los mayores de 60 años, indicando que el ensayo de Fase III evidenció la seguridad y la eficacia de la vacuna y no registró eventos adversos inesperados o graves relacionados al producto (Télam, 2021). Esta decisión significa una ampliación de la capacidad de vacunación de Argentina y un estrechamiento de cooperación sanitaria entre los dos Estados.

\section{Conclusiones}

A finales de marzo de 2020, China y Argentina concretaron una cooperación binacional en materia de salud. A partir de aquel entonces, en este marco cooperativo, los dos países han desarrollado diversos esfuerzos conjuntos para elevar la capacidad de gobernanza sanitaria de Argentina frente a la propagación del coronavirus.

La cooperación en gobernanza sanitaria entre China y Argentina se caracteriza por un predomino de los actores estatales y subestatales en la primera etapa, en la que la cooperación tuvo lugar principalmente a nivel gubernamental, especialmente en la provisión de materiales sanitarios, mientras que, con excepción de las empresas multinacionales, la participación de los actores no estatales se concentró mayoritariamente en los intercambios de experiencias antiepidémicas a través de los encuentros virtuales organizados por los Gobiernos centrales y locales. A su vez, la segunda etapa de la cooperación sanitaria cuenta con una mayor participación de los actores no estatales en varios aspectos. Las asociaciones mercantiles de origen chino y las comunidades chinas dentro del territorio argentino han jugado un rol complementario a los actores estatales y subestatales en el suministro de recursos necesarios, y son capaces de responder de manera inmediata y adecuada a las demandas locales ante la epidemia. Por su parte, las empresas farmacéuticas, organizaciones sin fines de lucro, como la Fundación Huésped, y los voluntarios contribuyeron con esfuerzos gigantescos al desarrollo de la investigación de vacunas. Al mismo tiempo, los Gobiernos centrales y locales de ambas naciones siguen profundizando las relaciones cooperativas en materia de salud, para enfrentarse conjuntamente con los desafíos que ha traído la pandemia.

A través de las dos etapas de cooperación, los dos países han cosechado varios logros en el combate de la COVID-19. En primer lugar, se ha aliviado en gran medida la falta de recursos sanitarios mediante la creación de un histórico puente aéreo entre ambas partes, que trasladó las compras argentinas y donaciones chinas en más de 40 operaciones especiales. En segundo lugar, las autoridades y el personal médico de Argentina han adquirido experiencias, conocimientos y aprendizajes mediante los intercambios en las videoconferencias, de modo que pueden estar más preparados y capacitados para afrontar la crisis sanitaria. En tercer lugar, se ha garantizado la seguridad del personal que lucha en la primera línea contra la enfermedad, incluidos los trabajadores de salud y los agentes policiales y militares, con la posesión de equipamientos de protección y el alto porcentaje de vacunación. Por último, estos resultados han ayudado a fortalecer el sistema de salud nacional de Argentina, de ahí que se ha visto reforzada la capacidad de gobernanza sanitaria de Argentina. En tal sentido, estos logros también han contribuido en gran medida a la CSS entre China y América Latina y el Caribe en el ámbito de la salud, defendiendo el interés del pueblo argentino y reduciendo la vulnerabilidad del país latinoamericano frente al coronavirus, y 
han impulsado la asociación de cooperación integral de China con esta región estableciendo un frente común para combatir la COVID-19.

No obstante, en la actualidad, Argentina sigue en medio de la pandemia y está encarando la segunda ola de epidemia. Con base en los éxitos anteriores obtenidos, más que nunca es necesaria la profundización en la cooperación entre China y Argentina en materia de salud y en otros ámbitos relacionados, así como una mayor participación de los actores no estatales para reunir diversos esfuerzos de la sociedad a fin de superar de manera unida la crisis sanitaria.

Más en concreto, ante el rápido crecimiento de contagios causado por la segunda ola de coronavirus, por una parte, hay que fortalecer la cooperación de provisión de insumos y equipos sanitarios críticos para seguir asegurando la protección a toda la sociedad contra el coronavirus y reforzando la capacidad del sistema de salud argentino. Por otra parte, se tiene que profundizar la cooperación en relación con las vacunas, para mantener su suministro y aumentar la cobertura de vacunación; mientras tanto hay que evitar la politización de las vacunas, fenómeno que hoy en día está ocurriendo en muchos países. Además, pese a que existen muchas diferencias entre China y Argentina con respecto al sistema político, el entorno cultural, la estructura social, entre otros aspectos, merece la pena intensificar el intercambio de experiencias y conocimientos entre los autoridades y profesionales para encontrar medidas tan efectivas como adecuadas a las condiciones propias de la sociedad argentina. Frente al gran impacto que la pandemia ha ejercido en la economía argentina, es necesario estrechar las relaciones comerciales entre ambos países, especialmente cuando China es el único país que ha reportado crecimiento económico durante el año pasado, para apaciguar las consecuencias negativas generadas por el coronavirus.

Por último, como hemos señalado, hay que dar importancia al rol de los actores no estatales en la gobernanza sanitaria, incentivando a toda la sociedad para que se comprometa a actuar en coordinación con los Gobierno central y subnacionales en los aspectos mencionados, y responder de forma cercana a las necesidades locales y comunitarias para prestar apoyo y servicios a las personas que más los necesitan. De tal forma, se logrará una efectiva gobernanza sanitaria desde múltiples niveles y se afianzará la construcción de la Ruta de la Seda Sanitaria en América Latina y el Caribe.

\section{Bibliografía}

Ámbito. (2021, febrero 12). El Gobierno aprobó el uso de emergencia de la vacuna de Sinopharm y el jueves llegarán un millón de dosis. Ámbito. https://www.ambito.com/politica/vacuna/el-gobierno-aprobo-el-uso-emergencia-la-sinopharm-y-el-jueves-llegaran-un-millon-dosis-n5171438

Arredondo, A., Orozco, E., Duperval, P., Cuadra, M., Cicero, M., Hernández, C., Zamudio, E., \& De Icaza, E. (2009). Temas Selectos en Sistemas de Salud. Costos, financiamiento, equidad y gobernanza, conceptos, tendencias y evidencias. Universidad Autónoma de Yucatan. Facultad de Ciencias Antropológicas. 
Ayerdi, R. (2020, mayo 27). 80 mil mascarillas y 30 mil guantes: La nueva donación del Gobierno chino. Perfil. https://www.perfil.com/noticias/politica/80-mil-mascarillas-30-mil-guantes-nueva-donacion-del-gobierno-chino.phtml

Cao, D. (2020, marzo 18). First medical teams return from Wuhan. China Daily. https://www.chinadaily.com.cn/a/202003/18/WS5e7193c5a31012821728009b.html

CNN. (2020, mayo 20). Médicos presentaron amparo en Argentina por la escasez de materiales de protección contra el coronavirus. CNN. https://edition.cnn.com/videos/spanish/2020/05/20/coronavirus-argentina-escasez-proteccion-material-epp-pkg-emilia-delfino-perspectivas-buenos-aires.cnn

Daniell, K. A., \& Kay, A. (2017). Multi-level Governance: An Introduction. En K. A. Daniell \& A. Kay (Eds.), Multi-level Governance: Conceptual challenges and case studies from Australia (1st ed., pp. 3-32). ANU Press. https://doi.org/10.22459/MG.11.2017.01

Diagonales. (2020, junio 17). Sistema sanitario bonaerense: La importancia y falta del recurso humano. Diagonales. Diagonales. https://diagonales.com/contenido/sistemasanitario-bonaerense-la-importancia-y-falta-del-recurso-humano/21100

Dinatale, M. (2020a, abril 9). El Gobierno enviará un avión especial a China para traer los insumos sanitarios donados por el COVID-19. Infobae. https://www.infobae.com/politica/2020/04/09/coronavirus-en-la-argentina-el-gobierno-enviara-un-avion-especial-a-china-para-traer-1500-respiradores-y-otros-insumos-sanitarios/

Dinatale, M. (2020b, junio 27). De barbijos a fondos para la investigación científica: La ayuda que la Argentina recibió del exterior para la lucha contra el COVID-19. Infobae. https://www.infobae.com/politica/2020/06/27/de-barbijos-a-fondos-para-lainvestigacion-cientifica-la-ayuda-que-la-argentina-recibio-del-exterior-para-lalucha-contra-el-covid-19/

Domínguez Martín, R. (2016). En los pliegues de la historia: Cooperación Sur-Sur y procesos de integración en América Latina y el Caribe. Estudos Internacionais: revista de relações internacionais da PUC Minas, 4 (2), 57-78.

https://doi.org/10.5752/P.2317-773X.2016v4n2p57-78

Domínguez Martín, R. (2017). La Princesa y el Dragón: Cooperación China en América Latina y más allá. Revista Internacional de Cooperación y Desarrollo, 4 (2), 3-27. https://doi.org/10.21500/23825014.3339

Dunford, M. (2020). Chinese and Development Assistance Committee (DAC) development cooperation and development finance: Implications for the BRI and international governance. Eurasian Geography and Economics, 61 (2), 125-136. https://doi.org/10.1080/15387216.2020.1716821

DW. (2021, febrero 22). Argentina autoriza uso de emergencia de vacuna de Sinopharm. DW. https://www.dw.com/es/argentina-autoriza-uso-de-emergencia-de-vacunade-sinopharm/a-56654987 
El Sol. (2020, julio 1). Importante donación de barbijos de China para el distrito. El Sol. https://elsolnoticias.com.ar/el-municipio-de-quilmes-recibio-una-importantedonacion-de-barbijos-de-china/

Esteban, P. (2020, septiembre 4). Coronavirus: "La terapia intensiva llegó al límite". Página 12. https://www.pagina12.com.ar/289739-coronavirus-la-terapia-intensivallego-al-limite

Fitz Patrick, M., \& Crucianelli, S. (2020, octubre 19). Coronavirus: ¿por qué Argentina llegó al millón de casos? Infobae. https://www.infobae.com/politica/2020/10/20/coronavirus-por-que-argentina-llego-al-millon-de-casos/

Fukuyama, F. (2013). What Is Governance? Governance, 26 (3), 347-368. https://doi.org/10.1111/gove.12035

Infobae. (2021, febrero 25). Llegó el avión de Aerolíneas Argentinas con 904 mil dosis de la vacuna china Sinopharm. Infobae. https://www.infobae.com/politica/2021/02/26/Ilego-el-avion-de-aerolineas-argentinas-con-904-mil-dosisde-la-vacuna-china-sinopharm/

Kaufman, D. (2005). 10 Myths about Governance and Corruption. Finance and Development, $42(3), 41-43$.

Kennan, G. F. (1971). Foreign Aid as a National Policy. Proceedings of the Academy of Political Science, 30 (3), 175. https://doi.org/10.2307/1173296

Keohane, R. O. (2005). After hegemony: Cooperation and discord in the world political economy (1st Princeton classic ed). Princeton University Press.

La Opinión Austral. (2020, abril 21). Autoridades sanitarias de Santa Cruz dialogaron con médicos de China por videoconferencia. La Opinión Austral. https://laopinionaustral.com.ar/localidades/autoridades-sanitarias-de-santa-cruz-dialogaron-conmedicos-de-china-por-videoconferencia-177195.html

Larre, A. (2020, junio 20). Argentina recibe material sanitario donado por China. Infodefensa. Recuperado. Infodefensa. https://www.infodefensa.com/latam/2020/06/20/noticia-argentina-recibe-material-sanitario-donadochina.html

La Vanguardia. (2020, marzo 10). Descienden a menos de 20 los nuevos casos confirmados de coronavirus en China. La Vanguardia. https://www.lavanguardia.com/vida/20200310/474077566482/descienden-casos-nuevos-coronavirus-china-positivos-contabilizados-video-seo-ext.html

Lewkowicz, J. (2021, abril 1). Aumentó la pobreza con el impacto de la pandemia. Página 12. https://www.pagina12.com.ar/333029-aumento-la-pobreza-con-el-impactode-la-pandemia

Marks, G. (1993). Structural policy and multilevel governance in the EC. En A. W. Cafruny \& G. G. Rosenthal (Eds.), The State of the European Community Vol. 2: The Maastricht Debates and Beyond (pp. 391-409). Lynne Rienne.

Mary, G. (2021, febrero 13). El Ejército Argentino recibe un hospital reubicable donado por 
China. Infodefensa. https://www.infodefensa.com/latam/2021/02/13/noticiallega-argentina-hospital-reubicable-procedente-china.html

Miranda, A. (2020, marzo 16). Son kits de detección del virus. Argentina recibirá ayuda sanitaria de China por coronavirus. Todojujuy. https://www.todojujuy.com/mundo/argentina-recibira-ayuda-sanitaria-china-coronavirusn132159

Morvaridi, B., \& Hughes, C. (2018). South-South Cooperation and Neoliberal Hegemony in a Post-aid World: South-South Cooperation in a Post-aid World. Development and Change, 49 (3), 867-892. https://doi.org/10.1111/dech.12405

NewsArgenChina. (2020a, noviembre 30). La Asociación Qingtian y el equipo de voluntarios chinos donaron materiales sanitarios en Once. NewsArgenChina. https://newsargenchina.com/html/show-1071.html

NewsArgenChina. (2020b, diciembre 5). El Equipo de Voluntarios Chinos en Argentina donó alimentos y materiales sanitarios a comedores de la Comuna 13. NewsArgenChina. https://newsargenchina.com/html/show-1081.html

Ng, G., \& Restivo, N. (2018, marzo 15). Comunidad china: Vivir en Argentina. Una aproximación a la experiencia de los inmigrantes chino. REDCAEM. https://chinayamericalatina.com/comunidad-china-vivir-en-argentina/

Niebieskikwiat, N. (2020, abril 13). Envío de Xi Jinping. Coronavirus en Argentina: Llegó un cargamento con donaciones chinas para el ministerio de Salud. Clarín. https://www.clarin.com/politica/coronavirus-argentina-llego-cargamento-donaciones-chinas-ministerio-salud_0_jILLWXGcO.html

Organización Mundial de la Salud. (2008). Informe sobre la salud en el mundo 2008: La atención primaria de salud, más necesaria que nunca. Organización Mundial de la Salud. https://apps.who.int/iris/handle/10665/43952

Ortiz, Z., Antonietti, L., Capriatti, A., Ramos, S., Romero, M., Mariani, J., Ortiz, F., \& Pecheny, M. (2020). Preocupaciones y demandas frente a COVID-19. Encuesta al personal de salud. MEDICINA (Buenos Aires), 80, 1-9.

Pérez Sarmenti, I. (2021, febrero 7). Argentina, destino de ocho ensayos clínicos de vacunas contra el covid-19. CNN en Español. https://cnnespanol.cnn.com/2021/02/07/argentina-destino-de-ocho-ensayos-clinicos-de-vacunas-contra-el-covid-19/

Prensa Gobierno de Santa Fe. (2020, abril 9). Perotti dialogó por videollamada con autoridades sanitarias de la provincia china de Jiangsu. Prensa Gobierno de Santa Fe. https://www.santafe.gob.ar/noticias/noticia/267309/

ProMendoza. (2020, mayo 9). La provincia hermana de Guangdong donó 60 mil barbijos a Mendoza. ProMendoza. https://www.promendoza.com/en/la-provincia-hermana-de-guangdong-dono-60-mil-barbijos-a-mendoza/

Rhodes, R. A. W. (2007). Understanding Governance: Ten Years On. Organization Studies, 28 (8), 1243-1264. https://doi.org/10.1177/0170840607076586 
Rosario Noticias. (2020a, junio 8). Rosario recibió una valiosa donación de insumos sanitarios del gobierno de Shanghai. Rosario Noticias. https://www.rosarionoticias.gob.ar/page/noticias/id/261392/title/Rosario-recibi\%C3\%B3-una-valiosadonaci\%C3\%B3n-de-insumos-sanitarios-del-gobierno-de-Shanghai

Rosario Noticias. (2020b, junio 29). Rosario recibió la donación de más de 21 mil kilos de alimentos por parte de la Cámara de Comercio Chino. Rosario Noticias. https://www.rosarionoticias.gob.ar/page/noticias/id/264645/title/Rosariorecibi\%C3\%B3-la-donaci\%C3\%B3n-de-m\%C3\%A1s-de-21-mil-kilos-de-alimentos-por-parte-de-la-C\%C3\%A1mara-de-Comercio-Chino-

Souza, A. de M. e (Ed.). (2014). Repensando a cooperação internacional para o desenvolvimento. IPEA.

Télam. (2019, agosto 2). China encabeza las exportaciones agrícolas argentinas con U\$S 1.500 millones. Télam. https://www.telam.com.ar/notas/201908/380958-china-encabeza-las-exportaciones-agricolas-argentinas-con-us-1500-millones.html

Télam. (2020a, junio 3). China desplazó a Brasil como principal socio comercial de la Argentina. Télam. https://www.telam.com.ar/notas/202006/471744-argentina-comercio-exterior-brasil-china.html

Télam. (2020b, junio 12). Argentina, en la lista de países que mejor respondieron a la pandemia según la revista Time. Télam. https://www.telam.com.ar/notas/202006/475590-argentina-paises-respuesta-pandemia-coronavirus-revista-time.html

Télam. (2021, marzo 26). Anmat autorizó la vacuna Sinopharm en los mayores de 60 años. Télam. https://www.telam.com.ar/notas/202103/548640-vizzotti-anuncio-laautorizacion-de-la-vacuna-sinopharm-para-adultos-mayores-de-60-anos.html

Vadell, J., Brutto, G. L., \& Leite, A. C. C. (2020). The Chinese South-South development cooperation: An assessment of its structural transformation. Revista Brasileira de Política Internacional, 63 (2), 1-22. https://doi.org/10.1590/00347329202000201

Xin, Q., \& Wen, S. (2020). China y la gobernanza sanitaria mundial desde la perspectiva de la "Ruta de la Seda Sanitaria". Relaciones internacionales contemporáneas, 6, 19-27.

Xinhua. (2020a, marzo 22). Expertos médicos chinos asesoran por videoconferencia a funcionarios de Argentina sobre experiencia de lucha contra COVID-19. Xinhuanet. http://spanish.xinhuanet.com/2020-03/22/c_138904220.htm

Xinhua. (2020b, abril 19). ESPECIAL: China y Argentina realizan histórico puente aéreo de suministros médicos para batalla contra COVID-19. Xinhuanet. http://spanish.xinhuanet.com/2020-04/19/c_138988841.htm

Xinhua. (2020c, julio 13). Dona provincia china de Guizhou insumos médicos a Argentina. Xinhuanet. http://spanish.xinhuanet.com/2020-07/13/c_139207495.htm 
Xinhua. (2020d, agosto 22). Argentina autoriza ensayos clínicos de fase III de vacuna inactivada china contra COVID-19. Xinhuanet. http://spanish.xinhuanet.com/202008/22/c_139309616.htm

Xinhua. (2020e, noviembre 8). ESPECIAL: Argentina renueva "puente aéreo" con China para contar con suministros para batalla contra COVID-19. Xinhuanet. http://spanish.xinhuanet.com/2020-11/08/c_139499331.htm

Zeng, A. (2021). Cooperación mundial en materia de gobernanza de la salud pública: Una perspectiva del desarrollo conjunto de China y África de la "Ruta de la Seda Sanitaria". Asia Occidental y África, 1, 26-47.

Zhang, J. (2020). Cooperación en materia de gobernanza de la salud pública entre China y el Sudeste Asiático: Un Estudio de caso de Anti-COVID-19. Estudios del Sudeste Asiático, 5, 24-42. 
